\title{
Three-dimensional buckled honeycomb boron lattice with vacancies as an intermediate phase on the transition pathway from $\alpha-B$ to $\gamma$-B
}

\author{
Woo Hyun Han ${ }^{1}$, Young Jun $\mathrm{Oh}^{2}$, Duk-Hyun Choe ${ }^{3}$, Sunghyun Kim ${ }^{1}$, In-Ho Lee ${ }^{4}$ and Kee Joo Chang ${ }^{1}$ \\ In the phase diagram of elemental boron, an unknown high-pressure form was identified as $\gamma$-orthorhombic boron $\left(\gamma\right.$ - $\left.B_{28}\right)$, \\ provoking studies of the extraordinary properties of its main building blocks, $B_{12}$ icosahedra and $B_{2}$ dumbbells. Although two \\ low-pressure phases, $\alpha$ - and $\beta$-rhombohedral boron $\left(\alpha-B_{12}\right.$ and $\left.\beta-B_{106}\right)$, are also composed of icosahedra, the detailed kinetics \\ and mechanisms of the structural transition from $\alpha-B_{12}$ or $\beta-B_{106}$ to $\gamma-B_{28}$ remain poorly understood. We report on new \\ metastable boron phases formed during the transition in high-pressure high-temperature conditions that were discovered using \\ the crystal structure search method. The metastable phases are understood to be a three-dimensional buckled defective \\ honeycomb lattice in which boron vacancies lead to a dynamically and mechanically stable structure with triangular motifs. \\ We suggest that the metastable phases act as intermediate states on the transition pathway from $\alpha-B_{12}$ to $\gamma-B_{28}$ owing to their \\ structural flexibility and low enthalpies, in the framework of Ostwald's step rule.
}

NPG Asia Materials (2017) 9, e400; doi:10.1038/am.2017.98; published online 7 July 2017

\section{INTRODUCTION}

Elemental boron exhibits a variety of allotropes and B-rich compounds owing to its electron deficiency compared with carbon that results in the ability to form multicenter bonds such as two- and three-center bonds. ${ }^{1,2}$ Among the three-dimensional (3D) polymorphs, two rhombohedral phases $\left(\alpha-B_{12}\right.$ and $\left.\beta-B_{106}\right)$ and a $\gamma$-orthorhombic phase $\left(\gamma-B_{28}\right)$ are known as pure boron allotropes with structural units called icosahedra $\left(B_{12}\right)$, and their crystal symmetries are determined by the arrangement of icosahedra building blocks. ${ }^{1}$ Although the $\alpha$ - and $\beta$-rhombohedral phases can be crystallized from amorphous $\mathrm{B},{ }^{3,4}$ their relative stability at ambient conditions is debated. There is a general consensus that although denser $\alpha-B_{12}$ is in a stable phase at elevated pressures, it transforms to $\beta-B_{106}$ as the temperature increases. ${ }^{5-7}$ However, based on extrapolation of the phase boundary, $\alpha-B_{12}$ has been claimed to be stable at normal conditions. ${ }^{8}$ Conversely, $\gamma$-orthorhombic boron is stable at high pressures and its crystal structure is now well established based on theoretical and experimental studies. ${ }^{5}$ Various experiments have demonstrated that $\gamma-\mathrm{B}_{28}$ can be synthesized from several starting materials, including amorphous boron, $\alpha-\mathrm{B}_{12}$, and $\beta-\mathrm{B}_{106}$, at pressures of $7-20 \mathrm{GPa}$ and temperatures of $1500-2500 \mathrm{~K} \cdot{ }^{5-9}$ Other boron phases such as $\alpha$-tetragonal $\left(\mathrm{T}-\mathrm{B}_{50}\right.$ or $\left.\mathrm{T}-\mathrm{B}_{52}\right)$ and $\beta$-tetragonal boron (T-192 or $\beta-B_{192}$ ) also contain icosahedra but have been suggested to be intermediate phases formed during structural transformations under high-pressure high-temperature conditions according to Ostwald's step rule. ${ }^{5,6,9,10}$

Although $\alpha-B_{12}$ and $\gamma-B_{28}$ contain distorted cubic close-packed $\mathrm{B}_{12}$ icosahedra, the structural difference is that $\gamma-\mathrm{B}_{28}$ has additional $\mathrm{B}_{2}$ dumbbells occupying all the octahedral cavities. The bonding characteristics of the icosahedra and $\mathrm{B}_{2}$ units are well studied in these two allotropes: two- and three-center bonds coexist within and between icosahedra, enabling icosahedra that satisfy electron counting rules and possess bond polarity attributed to the spatial asymmetry of the charge densities in three-center bonds. ${ }^{11-13}$ In $\gamma-B_{28}$, despite the predominantly covalent bonding nature between the $\mathrm{B}$ atoms, there is a partial charge transfer between the $B_{2}$ units and the $\mathrm{B}_{12}$ icosahedra in a NaCl-type structure, resulting in polar covalent bonds. ${ }^{5}$ The partial ionicity of $\gamma-\mathrm{B}_{28}$ is supported by experimental measurement of the electron density distribution ${ }^{13}$ and explains the pressure dependence of the band gap, which is less than that of covalent $\alpha-B_{12}$, and the transverse optical-longitudinal optical phonon splitting, which commonly occurs in polar materials. ${ }^{5,14,15}$ Because $B_{2}$ units are absent in the $\alpha-B_{12}$ phase, the mechanism for the pressure-induced transformation from $\alpha-B_{12}$ to $\gamma-B_{28}$ is not well understood. The $\alpha-B_{12}$ and $\gamma-B_{28}$ phases have different numbers of atoms per unit cell, necessitating a large supercell size to describe realistically the phase transition in theoretical simulations. Despite such difficulties, a few studies have attempted to explain the

${ }^{1}$ Department of Physics, Korea Advanced Institute of Science and Technology, Daejeon, Republic of Korea; ${ }^{2}$ Department of Materials Science and Engineering, University of Texas at Dallas, Richardson, TX, USA; ${ }^{3}$ Department of Physics, Applied Physics, and Astronomy, Rensselaer Polytechnic Institute, Troy, NY, USA and ${ }^{4}$ Korea Research Institute of Standards and Science, Daejeon, Republic of Korea

Correspondence: Professor KJ Chang, Department of Physics, Korea Advanced Institute of Science and Technology, Daehak-ro 291, Yuseong-gu, Daejeon 34141, Republic of Korea.

E-mail: kjchang@kaist.ac.kr

Received 1 February 2017; revised 21 April 2017; accepted 24 April 2017 
a

$$
\text { hex- } \mathrm{B}_{0}\left(P 6_{3} / m m c\right)
$$

$$
\text { hex- } \mathrm{B}_{1 / 8}(C 2 / m)
$$

Top view
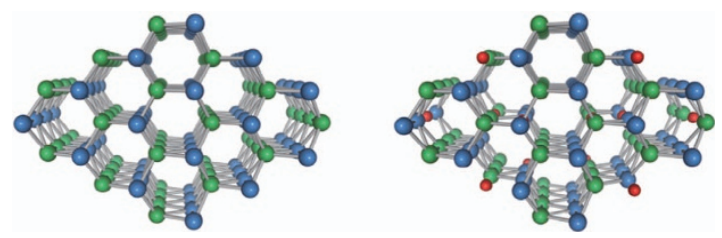

Side view
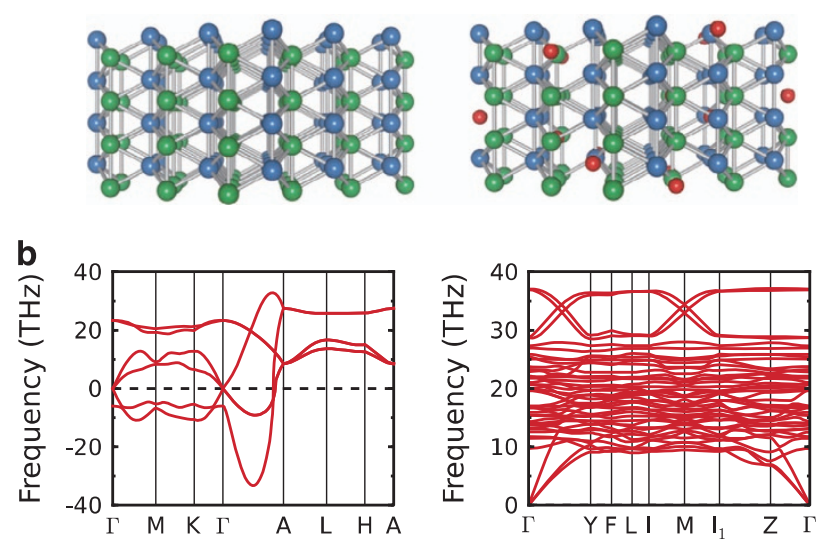

Figure 1 Structures and vibrational spectra of three-dimensional hexagonal boron. (a) Top and side views of the atomic structures of the hex- $B_{0}$ and hex- $B_{1 / 8}$ allotropes in the three-dimensional buckled honeycomb lattice, which have $P \sigma_{3} / \mathrm{mmc}$ and $C 2 / \mathrm{m}$ space groups, respectively. The blue and green circles represent the $B$ atoms with different heights in each buckled layer, and red circles indicate $B$ vacancies in the hex- $B_{1 / 8}$ allotrope. (b) The phonon spectra of the hex- $\mathrm{B}_{0}$ and hex- $\mathrm{B}_{1 / 8}$ allotropes at zero pressure.

transition mechanism between the two allotropes. First-principles calculations suggested that there may be three new boron phases, which are induced from $\gamma-B_{28}$ under deformation, on the transition pathway from $\alpha-B_{12}$ to $\gamma-B_{28} .{ }^{16}$ However, to match the number of atoms with that of $\gamma-B_{28}$, additional boron atoms were intentionally inserted into $\alpha-B_{12}$. Our understanding of the kinetic pathway to $\gamma-B_{28}$ is lacking, and the existence of metastable intermediate phases remains an open question.

In this work, we report the discovery of metastable boron phases, which appear in the energy landscape at high pressures, using an $a b$ initio evolutionary crystal structure search method. In metastable allotropes, icosahedra are completely broken, resulting in a $3 \mathrm{D}$ buckled honeycomb lattice with triangular motifs and $\mathrm{B}$ vacancies. With a certain fraction of $\mathrm{B}$ vacancies, the metastable structures become dynamically and mechanically stable, but are otherwise unstable. First-principles molecular dynamics (MD) simulations show that $B$ vacancies are mobile under extreme conditions such as high pressures and high temperatures, indicating that various metastable phases can exist depending on the concentration of $\mathrm{B}$ vacancies. Based on the results, we provide a microscopic description of the transition pathway from $\alpha-B_{12}$ to $\gamma-B_{28}$ in which metastable phases act as intermediate states during the structural transition in the framework of Ostwald's step rule.

\section{MATERIALS AND METHODS}

The enthalpy minimization and electronic structure calculations were performed within the framework of density functional theory. We used the functional form proposed by Perdew, Burke, and Ernzerhof for the exchange-correlation potential ${ }^{17}$ and the projector augmented wave potentials, ${ }^{18}$ as implemented in the VASP (Vienna $A b$ initio Simulation Package) code. ${ }^{19}$ A plane wave basis set was used to expand the wave functions with an energy cutoff of $600 \mathrm{eV}$. For Brillouin zone integration, a $k$-point set was generated with a grid spacing of $0.2 \AA^{-1}$. The ionic coordinates were fully optimized until the residual forces were $<0.01 \mathrm{eV} \AA^{-1}$. To obtain the phonon vibrational frequencies at arbitrary wave vectors, the dynamical matrices were calculated using a finite difference method, as implemented in the Phonopy software. ${ }^{20}$ We used a generalized solid-state climbing image nudged elastic band (CI-NEB) method, ${ }^{21,22}$ which is a modified version of the nudged elastic band method, to determine the transition pathways and energy barriers in the solid-solid transformations. For first-principles constant pressure-temperature MD simulations, we used the method of Parrinello and Rahman ${ }^{23}$ as implemented in the VASP code in which the temperature is controlled by a Langevin thermostat ${ }^{24}$ with an increasing energy cutoff up to $800 \mathrm{eV}$ to eliminate the Pulay stress and a time step of $1 \mathrm{fs}$ was selected.

\section{RESULTS AND DISCUSSION}

To search for new crystal structures of elemental B under pressure, we used an $a b$ initio evolutionary crystal structure search method, as implemented in the AMADEUS code..$^{25}$ This method is based on a combination of the conformational space annealing algorithm for global optimization with first-principles density functional calculations. The conformational space annealing algorithm uses three aspects: local energy/enthalpy minimization, a genetic algorithm to generate daughter solutions and simulated annealing to measure the similarities or differences between two solutions. This computational search algorithm is very efficient to search for stable and metastable structures because it maintains a population consisting of distinct low-enthalpy configurations in each generation. Thus, the conformational space annealing algorithm is similar to the current version of USPEX. ${ }^{26,27}$ The efficiency of the AMADEUS code has been demonstrated by successful applications for exploring $\mathrm{Si}$ and $\mathrm{C}$ allotropes with direct band gaps. ${ }^{28-30}$ For a system with $28 \mathrm{~B}$ atoms per unit cell, we found that the $\gamma-B_{28}$ structure containing $\mathrm{B}_{12}$ icosahedra is the most stable configuration at $40 \mathrm{GPa}$ pressure (see Supplementary Figure S1), in agreement with a previous result based on another evolutionary crystal search algorithm, USPEX. ${ }^{5}$ In addition, we found a new B allotrope with the second-lowest enthalpy structure, which has 14 atoms in the primitive cell with the $C 2 / m$ space group (Figure 1a).

The metastable $C 2 / m$ allotrope contains no $B_{12}$ icosahedra, in contrast to $\alpha-B_{12}$ and $\gamma-B_{28}$, and exhibits a metallic band structure (see Supplementary Figure S2). The crystal structure of the $\mathrm{C} 2 / \mathrm{m}$ allotrope is composed of buckled hexagonal layers and B vacancies. In the absence of $B$ vacancies, the buckled layers are simply stacked without lateral displacements, forming a 3D hexagonal lattice with the $P 6_{3} / m m c$ space group (Figure 1a). Each layer has a buckled honeycomb geometry, similar to monolayer silicene, ${ }^{31}$ but with more significant buckling. Owing to strong interlayer bonding, the B atoms have an eightfold coordination with similar intra- and interlayer bond lengths, leading to triangular motifs. Although the hypothetical $\mathrm{Pb}_{3} / \mathrm{mmc}$ structure is unstable because it violates the electron counting rules, it turns into a dynamically stable structure by introducing $\mathrm{B}$ vacancies, similar to the two-dimensional (2D) $\alpha$-sheet. ${ }^{32}$ In the $\mathrm{C} 2 / \mathrm{m}$ phase, two $\mathrm{B}$ atoms in the unit cell that corresponds to eight primitive cells in the $P 6_{3} / m m c$ structure are missing, resulting in a vacancy concentration of $\nu=1 / 8$. The optimized structural parameters for the $P 6_{3} / \mathrm{mmc}$ and $\mathrm{C} 2 / \mathrm{m}$ structures are summarized in Supplementary Table 1 . Recently, two metastable B allotropes with the $P 2_{1} / c$ and $C 2 / c$ space groups, which do not contain $B_{12}$ units, were 

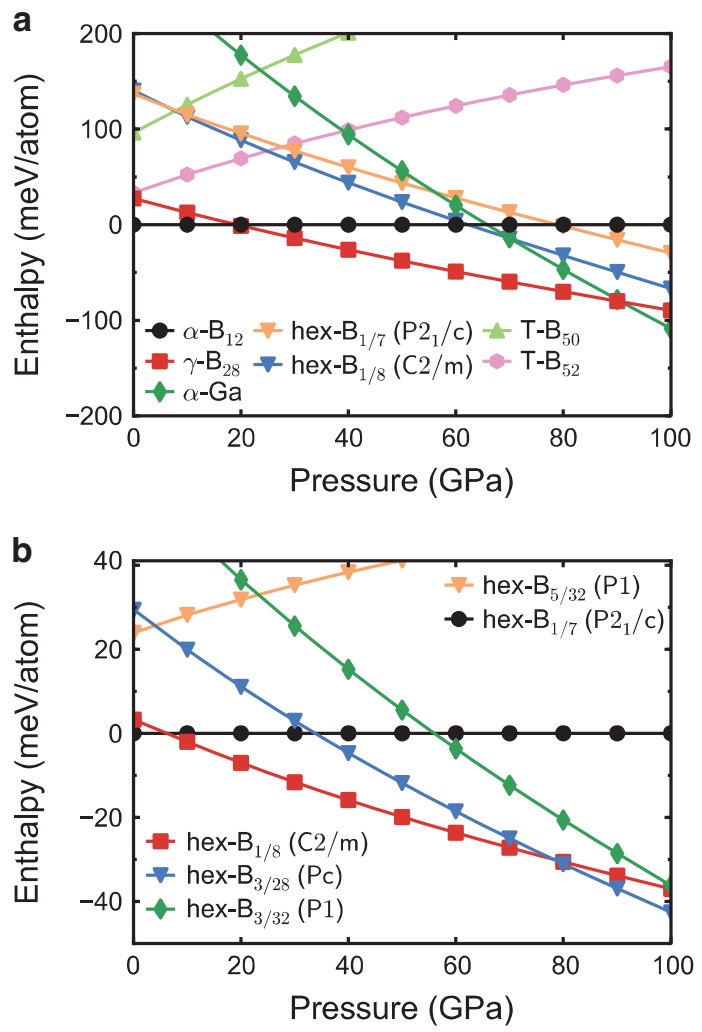

Figure 2 Enthalpies of various B allotropes. (a) The relative enthalpies of various B allotropes (see Supplementary Figure 4) plotted as a function of pressure. (b) The relative enthalpies of five hex- $B_{\nu}$ allotropes with different concentrations of $B$ vacancies, $\nu=5 / 32,1 / 7,1 / 8,3 / 28$ and $3 / 32$, as a function of pressure.

reported, ${ }^{33,34}$ and their concentrations of $\mathrm{B}$ vacancies were $\nu=1 / 7$ and $1 / 9$, respectively. To describe different vacancy concentrations, we use hex- $\mathrm{B}_{\nu}$ notation such as hex- $\mathrm{B}_{0}$, hex- $\mathrm{B}_{1 / 9}$, hex- $\mathrm{B}_{1 / 8}$ and hex- $\mathrm{B}_{1 / 7}$ for allotropes with the $P 6_{3} / m m c, C 2 / c, C 2 / m$ and $P 2_{1} / c$ space groups, respectively, in the $3 \mathrm{D}$ hexagonal lattice. The classification parameter $\nu$ is analogous to the hexagonal hole density $\eta$ used to distinguish various planar forms from 2D triangular lattice. $^{32}$ In 3D allotropes, we find that vacancies tend to disperse rather than aggregate, forming various configurations with different space groups.

To investigate the dynamical stability of hex- $\mathrm{B}_{v}$, we examined the full phonon spectrum. It is known that a flat triangular boron lattice is unstable with respect to a buckled shape owing to occupation of the antibonding states from three-center bonds. ${ }^{32}$ When hexagonal holes are introduced in the $2 \mathrm{D}$ triangular lattice, for example, in the boron $\alpha$-sheet, the lattice stability is greatly enhanced because hexagonal holes embedded in the triangular lattice serve as scavengers of extra electrons, giving rise to delocalized $\pi$-bonds near the holes instead of three-center bonds. ${ }^{35}$ Conversely, honeycomb and Kagome lattices are unstable owing to the lack of electrons from excess holes. In $2 \mathrm{D}$ metal-B systems such as $\mathrm{MoB}_{4}{ }^{36}$ $\mathrm{TiB}_{2}{ }^{37}$ and $\mathrm{MgB}_{6},{ }^{38}$ the electron transfer from metal ions to $\mathrm{B}$ networks balances the number of electrons, stabilizing the honeycomb and Kagome lattices. Similarly, the stability of $3 \mathrm{D}$ hex- $\mathrm{B}_{v}$ allotropes can be understood as a $3 \mathrm{D}$ extension of the $2 \mathrm{D}$ triangular lattice. Notably, B vacancies in hex- $\mathrm{B}_{\nu}$ allotropes act as acceptors similar to hexagonal holes in the $\alpha$-sheet and allow for balancing of a
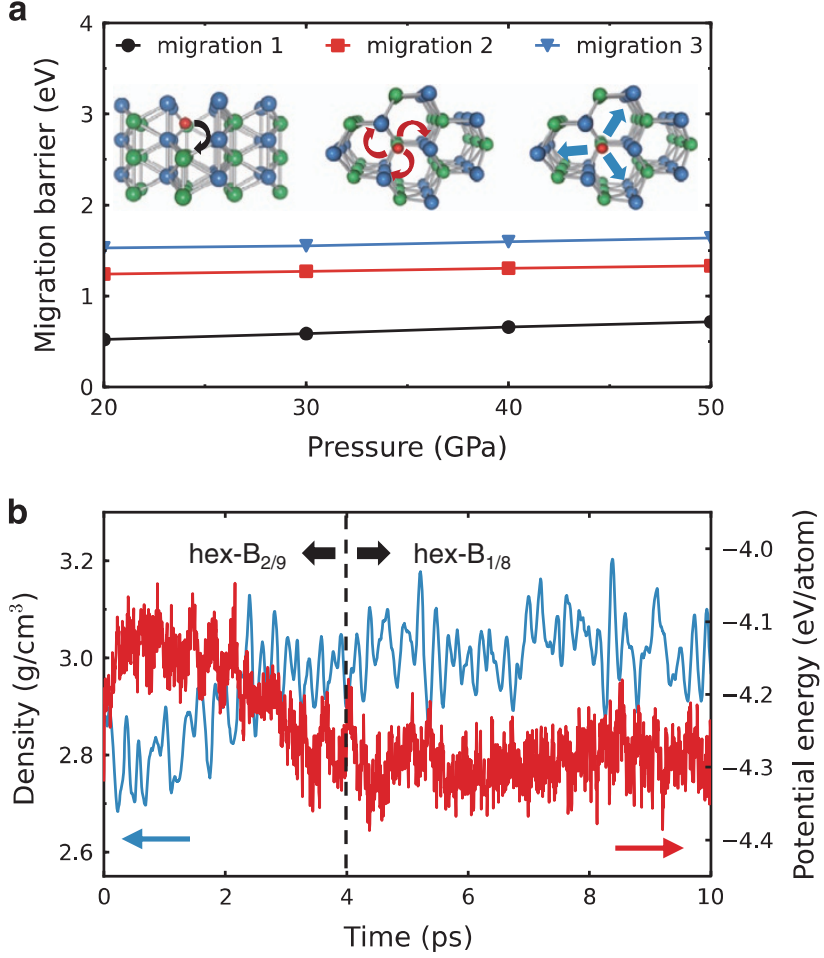

Figure 3 Migration of B vacancy. (a) The migration barriers for B-vacancy as a function of pressure for three different pathways. Boron vacancies are indicated by red circles, and the arrows represent their moving directions. (b) The fluctuations of potential energy and density during first-principles molecular dynamics (MD) simulations plotted for the hex- $B_{2 / 9}$ allotrope at $50 \mathrm{GPa}$ and $1500 \mathrm{~K}$. The vertical dotted line represents the simulation time when a structural transition occurs from hex- $B_{2 / 9}$ to hex- $B_{1 / 8}$ during constant pressure-temperature MD simulations.

the number of electrons, inducing delocalized $\pi$-bonds. For the hex- $\mathrm{B}_{1 / 8}$ and hex- $\mathrm{B}_{1 / 7}$ allotropes with the $C 2 / m$ and $P 2_{1} / c$ space groups, no imaginary phonon modes were found (Figure $1 b$ ), indicating that these structures are dynamically stable. Conversely, the hex- $\mathrm{B}_{0}$ structure without vacancies suffers from extra electrons and exhibits imaginary phonon modes (Figure 1b). Notably, as hole carriers are added to the hex- $\mathrm{B}_{0}$ structure, the imaginary phonon modes disappear, confirming that $\mathrm{B}$ vacancies have a role in dynamical stability (see Supplementary Figure S3). In addition to the dynamical properties, we investigated the mechanical properties of the $\mathrm{C} 2 / \mathrm{m}$ and $P 2{ }_{1} / \mathrm{c}$ structures by calculating the elastic constants (see Supplementary Table S2). Using stress-strain relations and Hill's formula, ${ }^{39,40}$ we note that the elastic constants satisfy mechanical stability criteria for the monoclinic cells of the $\mathrm{C} 2 / \mathrm{m}$ and $\mathrm{P}_{2} / \mathrm{c}$ structures. Based on the results, it is inferred that the metastable $C 2 / m$ and $P 2_{1} / c$ structures are likely formed under high pressure.

The relative enthalpies of hex- $\mathrm{B}_{\nu}$ and other boron allotropes (see Supplementary Figure S4) are plotted as a function of pressure in Figure 2a. The $\alpha-\mathrm{B}_{12}$ phase first transforms to $\gamma-\mathrm{B}_{28}$ at $19 \mathrm{GPa}$ and then to $\alpha$-Ga-type $B$ at $90 \mathrm{GPa}$, in agreement with previous calculations. $^{5,41}$ Near the transition pressure of $19 \mathrm{GPa}$, the enthalpy of the hex- $B_{1 / 8}$ structure is $\sim 100 \mathrm{meVper}$ atom higher than those of $\alpha-B_{12}$ and $\gamma-B_{28}$. However, the hex- $B_{1 / 8}$ and hex- $B_{1 / 7}$ allotropes become more stable than the $\alpha-B_{12}$ phase as the pressure increases above 62 and $80 \mathrm{GPa}$, respectively (Figure 2a). Conversely, although the hex- $\mathrm{B}_{1 / 7}$ structure has $3 \mathrm{meV}$ per atom lower energy 
a

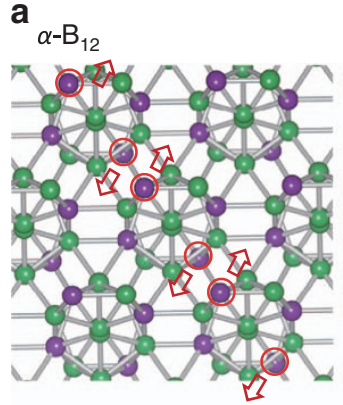

Transition state A

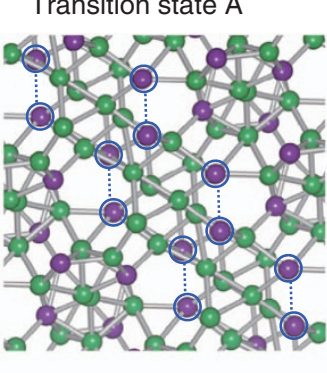

b $_{\text {hex- }} \mathrm{B}_{1 / 8}$
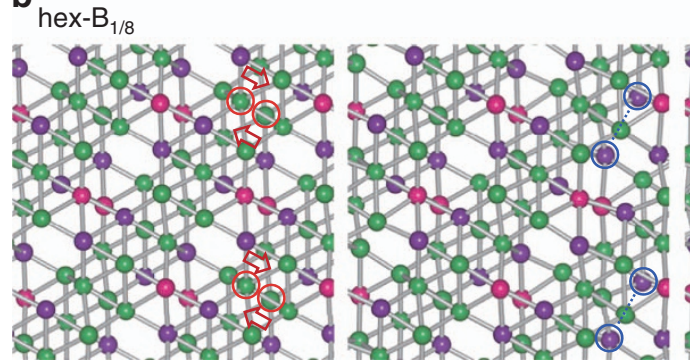
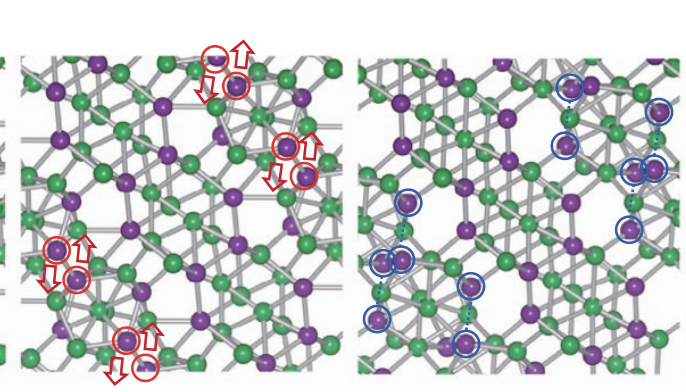

hex- $B_{1 / 7}$

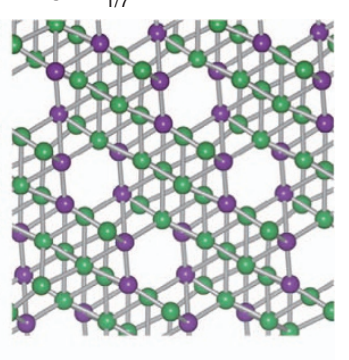

Transition state $\mathrm{C}$

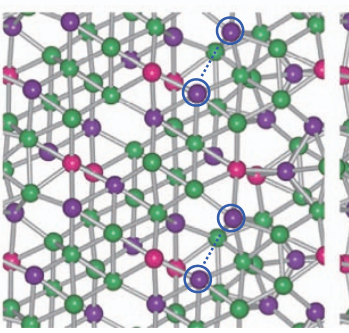

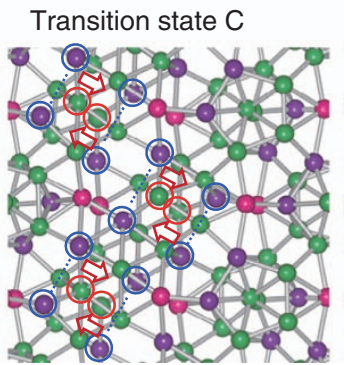

$\gamma-B_{28}$

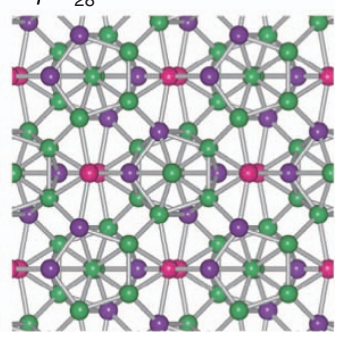

Figure 4 Transition pathways from $\alpha-B_{12}$ to $\gamma-B_{28}$. The atomic structures during the stepwise transformations (a) from $\alpha-B_{12}$ to hex- $B_{1 / 7}$ and (b) from hex- $B_{1 / 8}$ to $\gamma$-B $B_{28}$, with the lowest enthalpy barriers ( $\alpha_{1}$ and $\gamma_{1}$ in Figure $5 b$ ) from the solid-state climbing image nudged elastic band (Cl-NEB) method. The purple circles denote the $\mathrm{B}$ atoms that undergo major movements. The red arrows represent the directions of atomic movements, and the blue dotted lines indicate the formation of new bonds when a configuration changes to the next configuration. The red circles in (b) represent $B_{2}$ dumbbells to be formed in $\gamma-B_{28}$.

than the hex- $\mathrm{B}_{1 / 8}$ structure at zero pressure, the hex- $\mathrm{B}_{1 / 8}$ structure becomes more stable at pressures $>6 \mathrm{GPa}$. In recent experiments, it was suggested that $\alpha$-tetragonal boron, $T-B_{50}$ or $T-B_{52}$, may act as an intermediate phase during the structural transition from $\beta-B_{106}$ to $\gamma-B_{28}{ }^{6,9}$ However, the relative enthalpies of $T-B_{50}$ and $T-B_{52}$ rapidly increase with increasing pressure, as shown in Figure 2a, indicating that the hex- $\mathrm{B}_{1 / 8}$ structure is more likely an intermediate phase.

To investigate a possible transition pathway, we assumed that all of the $B_{12}$ icosahedra in $\alpha-B_{12}$ are broken during the structural transformation. Then, the intermediate structure can be represented by an arrangement of $\mathrm{B}$ vacancies in the hex- $\mathrm{B}_{0}$ structure. For two supercells containing 28 and 32 atoms in the hex- $\mathrm{B}_{0}$ structure, we considered various concentrations of $\mathrm{B}$ vacancies, such as $\nu=3 / 32$, $3 / 28,4 / 32 \quad(=1 / 8), 4 / 28 \quad(=1 / 7)$ and $5 / 32$. For each vacancy concentration, we searched for the lowest enthalpy configuration of $B$ vacancies at zero pressure using the AMADEUS protocol and examined the relative stability of five hex- $B_{\nu}$ structures under pressure, as illustrated in Figure $2 \mathrm{~b}$. Among the hex $-\mathrm{B}_{\nu}$ allotropes, the hex- $\mathrm{B}_{1 / 7}$ allotrope had the lowest enthalpy for low pressures $<6 \mathrm{GPa}$. In the pressure range of $6-80 \mathrm{GPa}$, the lowest enthalpy structure is the hex- $\mathrm{B}_{1 / 8}$ allotrope. As the pressure increases above $80 \mathrm{GPa}$, the $\mathrm{B}$ allotropes with low vacancy concentrations such as hex- $\mathrm{B}_{3 / 28}$ and hex- $\mathrm{B}_{3 / 32}$ become more stable. Thus, there is a tendency that denser structures with lower vacancy concentrations are preferred as the pressure increases.

Because the type of hex- $\mathrm{B}_{\nu}$ allotrope depends on the vacancy concentration, the migration of $\mathrm{B}$ vacancies strongly affects their structural stability. We calculated the B-vacancy migration barrier using a solid-state CI-NEB method. ${ }^{21,22}$ For the hex- $\mathrm{B}_{1 / 8}$ structure, which has four vacancies in an orthorhombic hex- $\mathrm{B}_{0}$ unit cell, we considered three migration pathways, as shown in Figure 3a. Along pathway 1, a vacancy hops from one buckled layer to its adjacent layer, whereas it migrates to the first and third nearest neighbor sites within a buckled layer, which are denoted as pathways 2 and 3, respectively. The migration barriers along the three pathways are plotted for pressures from 20 to $50 \mathrm{GPa}$. Along pathways 1, 2 and 3, the migration barriers at $20 \mathrm{GPa}$ are estimated to be $0.52,1.24$ and $1.53 \mathrm{eV}$, respectively. Although the interlayer hopping is relatively easier, it is expected that vacancy diffusion within the buckled layer also occurs at elevated temperatures. When $\gamma-B_{28}$ is synthesized from amorphous boron, $\alpha-B_{12}$ and $\beta-B_{106}$, extreme conditions such as high pressures and high temperatures are usually adopted. ${ }^{5-9}$ We examined the thermal motions of atoms in the hex- $\mathrm{B}_{1 / 8}$ structure by calculating the mean squared-displacements, defined as $<R^{2}(t)>=\left(\frac{1}{N}\right) \sum_{i=1}^{N}\left|\vec{R}_{i}(t)-\vec{R}_{i}(0)\right|^{2}$, through firstprinciples MD simulations at 1500 and $2200 \mathrm{~K}$. For the $\gamma-\mathrm{B}_{28}$ phase, which was taken as a reference structure for comparison, we found almost no fluctuations in mean-squared displacement at $2200 \mathrm{~K}$ (see Supplementary Figure S5). In the case of hex- $\mathrm{B}_{1 / 8}$, although the mean-squared displacements are $<0.5 \AA^{2}$ at $1500 \mathrm{~K}$, they significantly increase to $4-6 \AA^{2}$ during $80 \mathrm{ps}$ at $2200 \mathrm{~K}$. The enhanced mean-squared displacements are largely accompanied by the migration of B atoms within the same buckled layer. Thus, our results support the general tendency of migration of B vacancies at high temperatures.

To examine the effects of pressure and temperature on the movement of vacancies, we performed constant-pressure MD simulations at 500 and $1500 \mathrm{~K}$. For the initial configuration, we chose the hex- $\mathrm{B}_{2 / 9}$ structure with a relatively high concentration of vacancies in which 8 vacancies are randomly distributed in the 36-atom orthorhombic unit cell of hex- $\mathrm{B}_{0}$. From the enthalpy-vacancyconcentration curves shown in Figure $2 \mathrm{~b}$, the initial structure is expected to transform to hex- $\mathrm{B}_{\nu}$ allotropes with lower vacancy concentrations as the pressure increases. At a low temperature of $500 \mathrm{~K}$, we found no structural change and only small fluctuations of 

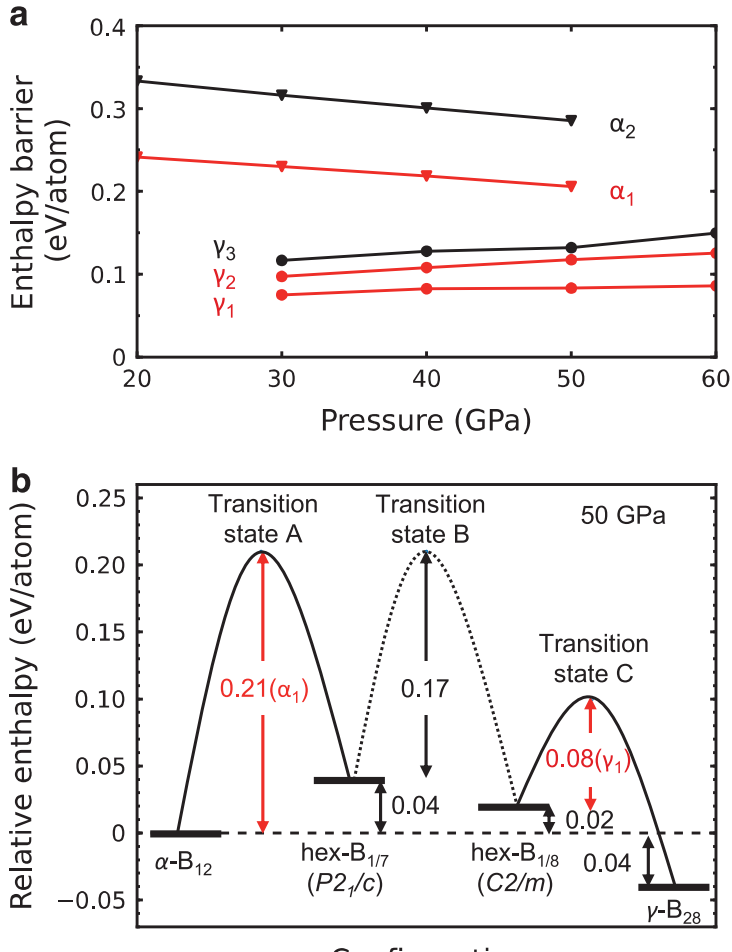

Configuration

Figure 5 Schematic diagram of the phase transition. (a) The enthalpy barriers as a function of pressure for various transition pathways from $\alpha-B_{12}$ to hex- $B_{1 / 7}\left(\alpha_{1}\right.$ and $\left.\alpha_{2}\right)$ and from hex-B $B_{1 / 8}$ to $\gamma-B_{28}\left(\gamma_{1}, \gamma_{2}\right.$ and $\left.\gamma_{3}\right)$, with the lowest enthalpy barriers for $\alpha_{1}$ and $\gamma_{1}$ in Figure 4. The stepwise and concerted transformations are represented by red $\left(\alpha_{1}, \gamma_{1}\right.$ and $\left.\gamma_{2}\right)$ and black $\left(\alpha_{2}\right.$ and $\left.\gamma_{3}\right)$ lines, respectively (see Supplementary Figure S7). (b) The enthalpy diagram for the transition from $\alpha-B_{12}$ to $\gamma-B_{28}$ at $50 \mathrm{GPa}$ with the hex- $B_{1 / 7}$ and hex- $B_{1 / 8}$ allotropes as intermediate phases in the framework of the Ostwald's step rule. The dotted line indicates the enthalpy barrier for the transition from hex- $B_{1 / 7}$ to hex- $B_{1 / 8}$, which is considered the upper bound because of the restricted supercell geometry (see Supplementary Figure S8).

the potential energy even when the pressure increased to $50 \mathrm{GPa}$ (see Supplementary Figure S6). Conversely, at $1500 \mathrm{~K}$, the potential fluctuations were greatly enhanced. At $40-50 \mathrm{GPa}$, the mass density significantly increased, accompanied by large potential fluctuations, indicating a reduction of the vacancy concentration (Figure $3 \mathrm{~b}$ ). During variable cell-shape MD simulations at 40 and $50 \mathrm{GPa}$, we found that the buckled planes were reoriented when vacancies migrated to form denser structures. Although the final configuration was not well identified due to thermal fluctuations, it is clear that $\mathrm{B}$ vacancies are very mobile at high pressures and high temperatures, thus the hex- $B_{\nu}$ structure without $B_{12}$ icosahedra is flexible, in contrast to $\alpha-B_{12}$ and $\gamma-B_{28}$.

Although both $\alpha-B_{12}$ and $\gamma-B_{28}$ consist of close-packed icosahedra, there is a structural discrepancy in that $\gamma-B_{28}$ has additional $B_{2}$ units inserted between icosahedra. During the transition from $\alpha-B_{12}$ to $\gamma-B_{28}$, it is difficult to explain the formation of the $B_{2}$ units if the icosahedra are preserved. To resolve this counting problem, it is necessary to introduce an intermediate phase that does not contain icosahedra. Therefore, we considered the hex- $\mathrm{B}_{\nu}$ allotrope to be the best candidate for the intermediate phase because of its low enthalpy and lack of icosahedra. Moreover, the number of B atoms per unit cell is flexible because the vacancies freely migrate under extreme conditions. Figure 4 shows a possible transition pathway from $\alpha-B_{12}$ to
$\gamma-B_{28}$ through two intermediate phases, hex- $B_{1 / 7}$ and hex- $B_{1 / 8}$, which was obtained using the solid-state CI-NEB method. The key features of this transition mechanism are that all of the icosahedra of $\alpha-B_{12}$ are broken, the intermediate hex- $B_{1 / 7}$ structure turns to the hex- $\mathrm{B}_{1 / 8}$ structure by rearrangement of the vacancies, and $\gamma-\mathrm{B}_{28}$, consisting of icosahedra and dumbbells, is formed. In the initial process, some atoms in the icosahedra undergo large lattice relaxations and rebonding with active atoms in adjacent icosahedra, as shown in Figure 4a. As the icosahedra are completely disintegrated, the intermediate hex- $\mathrm{B}_{1 / 7}$ structure is formed, maintaining the same number of atoms per unit cell as that of $\alpha-B_{12}$. As the vacancies are rearranged, the hex- $B_{1 / 7}$ structure immediately turns to the denser hex- $B_{1 / 8}$ structure with a lower enthalpy, as demonstrated by MD simulations (Figure 3). To visualize the formation of $\gamma-B_{28}$, we chose a 28 -atom orthorhombic cell for the hex- $\mathrm{B}_{1 / 8}$ structure, as shown in Figure $4 \mathrm{~b}$. In the final process, the $\mathrm{B}$ atoms in dense regions are reconnected to each other and reform icosahedra and those in vacancy channels remain as dumbbells between the newly formed icosahedra.

During the transition from $\alpha-B_{12}$ to hex- $B_{1 / 7}$, we considered two different transition pathways: (i) a concerted transformation in which all the icosahedra in $\alpha-B_{12}$ are simultaneously broken via relaxations, and (ii) a stepwise transformation in which bond-breaking relaxations first occur for half of the icosahedra and are proceeded by the other half (see Supplementary Figure S7). Similar concerted and stepwise transformations are possible for the transition from hex- $\mathrm{B}_{1 / 8}$ to $\gamma-B_{28}$. In Figure 5a, the enthalpy barriers are plotted as a function of pressure for the $\alpha-B_{12} \rightarrow$ hex $-B_{1 / 7}$ and hex $-B_{1 / 8} \rightarrow \gamma-B_{28}$ transitions. We found that the enthalpy barriers are lower for the stepwise transitions than for the concerted transitions. In addition, we note that the enthalpy barriers for $\alpha-B_{12} \rightarrow$ hex- $B_{1 / 7}$ are higher than those of hex- $B_{1 / 8} \rightarrow \gamma-B_{28}$, indicating that it is more difficult to break than to assemble the icosahedra. The calculated enthalpy barriers for the $\alpha-B_{12} \rightarrow$ hex- $B_{1 / 7}$ transition were $<.25 \mathrm{eV}$ per atom at $20-50 \mathrm{GPa}$. In previous theoretical calculations for the graphite-to-diamond phase transition, ${ }^{42}$ the enthalpy barriers for the nucleation process were lower than those for the concerted pathways. Similarly, for boron allotropes, the enthalpy barrier will be lower if the transition occurs via nucleation. In the nucleation process, the hex- $B_{1 / 7}$ and hex- $\mathrm{B}_{1 / 8}$ allotropes are still expected to appear because icosahedra are likely to be disintegrated at high-pressure high-temperature conditions.

Figure $5 \mathrm{~b}$ illustrates a schematic enthalpy diagram for the transition pathway from $\alpha-\mathrm{B}_{12}$ to $\gamma-\mathrm{B}_{28}$ under pressure. In $\alpha-\mathrm{B}_{12}$, because the interactions between icosahedra become stronger as the pressure increases, the icosahedra are vulnerable to bond-breaking relaxations. The enthalpy barrier for the transition from $\alpha-B_{12}$ to hex- $B_{1 / 7}$ is slightly reduced from 0.25 to $0.21 \mathrm{eV}$ per atom as the pressure increases from 20 to $50 \mathrm{GPa}$. This tendency agrees with the experimental observation that the kinetic barrier between $\beta-\mathrm{B}_{106}$ and $\gamma-B_{28}$ decreases with increasing pressure. ${ }^{9}$ Once hex- $B_{1 / 7}$ is formed after the icosahedra are broken, it immediately transforms to the hex- $\mathrm{B}_{1 / 8}$ structure with lower enthalpy. To clarify the transition pathway between the hex- $B_{1 / 7}$ and hex- $B_{1 / 8}$ structures, we used the solid-state CI-NEB method for a supercell with 84 atoms, the least common multiple of 12 and 28 that can describe both densities of $\nu=1 / 7$ and $1 / 8$. As vacancies migrate, the buckled hexagonal layers are reoriented and the density of vacancies changes from $\nu=1 / 7$ to $1 / 8$. In the NEB images along the pathway, the hex- $\mathrm{B}_{1 / 7}$ and hex- $\mathrm{B}_{1 / 8}$ structures coexist with grain boundaries (see Supplementary Figure S8). The enthalpy barrier for the hex- $\mathrm{B}_{1 / 7} \rightarrow$ hex $-\mathrm{B}_{1 / 8}$ transition 
was calculated to be $0.17 \mathrm{eV}$ per atom at $50 \mathrm{GPa}$. This enthalpy barrier is considered the upper bound because atomic arrangements are restricted within the supercell elongated along one direction and only the concerted motions are considered rather than the nucleation process. A large supercell containing thousands of atoms may avoid such restrictions, but is far beyond the current first-principles calculation limit. Despite the restricted cell geometry, the transition pathway from hex $-B_{1 / 7}$ to hex- $B_{1 / 8}$ is obtained via reorientation of the buckled hexagonal planes without significant atomic rearrangements. At the final stage, icosahedra are reformed in the hex- $B_{1 / 8}$ structure and extra $B$ atoms remain as $B_{2}$ dumbbells. In the transition from hex- $B_{1 / 8}$ to $\gamma$ - $B_{28}$, the enthalpy barrier is only $0.08 \mathrm{eV}$ per atom at $50 \mathrm{GPa}$. Based on the results, it is inferred that the metastable hex- $B_{1 / 7}$ and hex- $B_{1 / 8}$ phases act as intermediate states in the transformation from $\alpha-B_{12}$ to $\gamma-B_{28}$ under extreme pressure and temperature conditions. The simulated $\mathrm{x}$-ray diffraction patterns of the metastable phases (see Supplementary Figure S9) can be used to identify unknown intermediate phases that may appear during structural transformations.

\section{CONCLUSION}

In summary, we predicted several metastable boron phases using the evolutionary crystal structure search method and suggest that two metastable allotropes with $C 2 / m$ and $P 2_{1} / c$ space groups have important roles as intermediate phases during the structural transformation from $\alpha-\mathrm{B}_{12}$ to $\gamma-\mathrm{B}_{28}$ at high pressures and high temperatures. The metastable phases, formed after the $\mathrm{B}_{12}$ icosahedra are disintegrated, are understood to be a three-dimensional buckled honeycomb lattice with triangular motifs and become dynamically and mechanically stable in the presence of B vacancies. The first-principles MD simulations showed that B vacancies are mobile at high pressures and high temperatures, indicating the structural flexibility of the metastable phases in contrast to other known allotropes containing icosahedra. In the framework of Ostwald's rule of stages, introduction of the metastable phases enables us to explain the mysterious origin of $B_{2}$ units as well as the different arrangement of icosahedra in $\gamma-B_{28}$. Our results provide insights into understanding the role of metastable $B$ phases during structural transformations and assist in revealing the mechanisms of other unexplained solid-solid transitions between B allotropes that contain icosahedra.

\section{CONFLICT OF INTEREST}

The authors declare no conflict of interest.

\section{ACKNOWLEDGEMENTS}

This work was supported by Samsung Science and Technology Foundation under Grant No. SSTFBA1401-08 and by the Brain Korea 21 PLUS Project of Korea Government.

Author contributions: WHH performed the theoretical calculations and data analysis and participated in manuscript preparation. All authors contributed to the scientific discussion. IHL performed the crystal structure search calculations, and SK calculated the $\mathrm{x}$-ray diffraction patterns. KJC conducted the research, performed data analysis and participated in manuscript preparation.

1 Albert, B. \& Hillebrecht, H. Boron: elementary challenge for experimenters and theoreticians. Angew. Chem. Int. Ed. 48, 8640-8668 (2009).

2 Li, X.-B., Xie, S.-Y., Zheng, H., Tian, W. Q. \& Sun, H.-B. Boron based two-dimensional crystals: theoretical design, realization proposal and applications. Nanoscale 7, 18863-18871 (2015).
3 Decker, B. \& Kasper, J. The crystal structure of a simple rhombohedral form of boron. Acta Crystallogr. 12, 503-506 (1959).

4 Hughes, R. E., Kennard, C. H. L., Sullenger, D. B., Weakliem, H. A., Sands, D. E. \& Hoard, J. L. The structure of $\beta$-rhombohedral boron. J. Am. Chem. Soc. 85, 361-362 (1963).

5 Oganov, A. R., Chen, J., Gatti, C., Ma, Y., Ma, Y., Glass, C. W., Liu, Z., Yu, T., Kurakevych, O. O. \& Solozhenko, V. L. Ionic high-pressure form of elemental boron. Nature 457, 863-867 (2009).

6 Kurakevych, O. O., Le Godec, Y., Hammouda, T. \& Goujon, C. Comparison of solid-state crystallization of boron polymorphs at ambient and high pressures. High Press. Res. 32 , 30-38 (2012).

7 Solozhenko, V. L. \& Kurakevych, O. O. Equilibrium $p-T$ phase diagram of boron: experimental study and thermodynamic analysis. Sci. Rep. 3, 2351 (2013).

8 Parakhonskiy, G., Dubrovinskaia, N., Bykova, E., Wirth, R. \& Dubrovinsky, L. Experimental pressure-temperature phase diagram of boron: resolving the longstanding enigma. Sci. Rep. 1, 96 (2011).

9 Qin, J., Irifune, T., Dekura, H., Ohfuji, H., Nishiyama, N., Lei, L. \& Shinmei, T. Phase relations in boron at pressures up to $18 \mathrm{GPa}$ and temperatures up to $2200^{\circ} \mathrm{C}$. Phys. Rev. B 85, 014107 (2012).

$10 \mathrm{Ma}$, Y., Prewitt, C. T., Zou, G., Mao, H.-k. \& Hemley, R. J. High-pressure high-temperature $\mathrm{x}$-ray diffraction of $\beta$-boron to $30 \mathrm{GPa}$. Phys. Rev. B 67, 174116 (2003).

11 Fujimori, M., Nakata, T., Nakayama, T., Nishibori, E., Kimura, K., Takata, M. \& Sakata, M. Peculiar covalent bonds in $\alpha$-rhombohedral boron. Phys. Rev. Lett. 82, 4452 (1999).

$12 \mathrm{He}$, J., Wu, E., Wang, H., Liu, R. \& Tian, Y. Ionicities of boron-boron bonds in $\mathrm{B}_{12}$ icosahedra. Phys. Rev. Lett. 94, 015504 (2005).

13 Mondal, S., van Smaalen, S., Schönleber, A., Filinchuk, Y., Chernyshov, D., Simak, S. I., Mikhaylushkin, A. S., Abrikosov, I. A., Zarechnaya, E., Dubrovinsky, L. \& Dubrovinskaia, N. Electron-deficient and polycenter bonds in the high-pressure $\gamma$-B $\mathrm{B}_{28}$ phase of boron. Phys. Rev. Lett. 106, 215502 (2011).

14 Oganov, A. R. \& Solozhenko, V. L. Boron: a hunt for superhard polymorphs. J. Superhard Mater. 31, 285-291 (2009).

15 Oganov, A. R., Solozhenko, V. L., Gatti, C., Kurakevych, O. O. \& Le Godec, Y. The high-pressure phase of boron, $\gamma$ - $B_{28}$ : disputes and conclusions of 5 years after discovery. J. Superhard Mater. 33, 363-379 (2011).

16 An, Q., Goddard III, W. A., Xiao, H. \& Cheng, T. Deformation induced solid-solid phase transitions in gamma boron. Chem. Mater. 26, 4289-4298 (2014).

17 Perdew, J. P., Burke, K. \& Ernzerhof, M. Generalized gradient approximation made simple. Phys. Rev. Lett. 77, 3865-3868 (1996).

18 Blöchl, P. E. Projector augmented-wave method. Phys. Rev. B 50, 17953-17979 (1994).

19 Kresse, G. \& Furthmüller, J. Efficient iterative schemes for ab initio total-energy calculations using a plane-wave basis set. Phys. Rev. B 54, 11169-11186 (1996).

20 Togo, A. \& Tanaka, I. First principles phonon calculations in materials science. Scr. Mater. 108, 1-5 (2015).

21 Henkelman, G., Uberuaga, B. P. \& Jónsson, H. A climbing image nudged elastic band method for finding saddle points and minimum energy paths. J. Chem. Phys. 113, 9901-9904 (2000)

22 Sheppard, D., Xiao, P., Chemelewski, W., Johnson, D. D. \& Henkelman, G. A generalized solid-state nudged elastic band method. J. Chem. Phys. 136, 074103 (2012).

23 Parrinello, M. \& Rahman, A. Polymorphic transitions in single crystals: a new molecular dynamics method. J. Appl. Phys. 52, 7182-7190 (1981).

24 Allen, M. P. \& Tildesley, D. J. Computer Simulation of Liquids (Oxford University Press, Oxford, 1989).

25 Lee, I.-H., Oh, Y. J., Kim, S., Lee, J. \& Chang, K. J. Ab initio materials design using conformational space annealing and its application to searching for direct band gap silicon crystals. Comput. Phys. Commun. 203, 110-121 (2016).

26 Oganov, A. R. \& Glass, C. W. Crystal structure prediction using ab initio evolutionary techniques: principles and applications. J. Chem. Phys. 124, 244704 (2006).

27 Lyakhov, A. O., Oganov, A. R., Stokes, H. T. \& Zhu, Q. New developments in evolutionary structure prediction algorithm USPEX. Comput. Phys. Commun. 184, 1172-1182 (2013)

28 Lee, I.-H., Lee, J., Oh, Y. J., Kim, S. \& Chang, K. J. Computational search for direct band gap silicon crystals. Phys. Rev. B 90, 115209 (2014).

29 Oh, Y. J., Lee, I.-H., Kim, S., Lee, J. \& Chang, K. J. Dipole-allowed direct band gap silicon superlattices. Sci. Rep. 5, 18086 (2015).

30 Oh, Y. J., Kim, S., Lee, I.-H., Lee, J. \& Chang, K. J. Direct band gap carbon superlattices with efficient optical transition. Phys. Rev. B 93, 085201 (2016).

31 Cahangirov, S., Topsakal, M., Aktürk, E., Şahin, H. \& Ciraci, S. Two- and one-dimensional honeycomb structures of silicon and germanium. Phys. Rev. Lett. 102, 236804 (2009).

32 Tang, H. \& Ismail-Beigi, S. Novel precursors for boron nanotubes: the competition of two-center and three-center bonding in boron sheets. Phys. Rev. Lett. 99, 115501 (2007).

33 Pickard, C. J. \& Needs, R. J. Ab initio random structure searching. J. Phys. Condens. Matter 23, 053201 (2011).

34 Zhu, Q., Oganov, A. R., Lyakhov, A. O. \& Yu, X. Generalized evolutionary metadynamics for sampling the energy landscapes and its applications. Phys. Rev. B 92, 024106 (2015). 
35 Galeev, T. R., Chen, Q., Guo, J.-C., Bai, H., Miao, C.-Q., Lu, H.-G., Sergeeva, A. P., Li, S.-D. \& Boldyrev, A. I. Deciphering the mystery of hexagon holes in an all-boron graphene $\alpha$-sheet. Phys. Chem. Chem. Phys. 13, 11575-11578 (2011).

36 Xie, S.-Y., Li, X.-B., Tian, W. Q., Chen, N.-K., Zhang, X.-L., Wang, Y., Zhang, S. \& Sun, H.-B. First-principles calculations of a robust two-dimensional boron honeycomb sandwiching a triangular molybdenum layer. Phys. Rev. B 90, 035447 (2014).

37 Zhang, L. Z., Wang, Z. F., Du, S. X., Gao, H.-J. \& Liu, F. Prediction of a Dirac state in monolayer $\mathrm{TiB}_{2}$. Phys. Rev. B 90, 161402 (2014).

38 Xie, S.-Y., Li, X.-B., Tian, W. Q., Chen, N.-K., Wang, Y., Zhang, S. \& Sun, H.-B. A nove two-dimensional $\mathrm{MgB}_{6}$ crystal: metal-layer stabilized boron kagome lattice. Phys. Chem. Chem. Phys. 17, 1093-1098 (2015).

39 Hill, R. The elastic behaviour of a crystalline aggregate. Proc. Phys. Soc 65 349-354 (1952).

40 Le Page, Y. \& Saxe, P. Symmetry-general least-squares extraction of elastic data for strained materials from ab initio calculations of stress. Phys. Rev. B 65 104104 (2002)

$41 \mathrm{He}$, C. \& Zhong, J. X. Structures, stability, mechanical and electronic properties of $\alpha$-boron and $\alpha^{*}$-boron. AIP Adv. 3, 042138 (2013).
42 Khaliullin, R. Z., Eshet, H., Kühne, T. D., Behler, J. \& Parrinello, M. Nucleation mechanism for the direct graphite-to-diamond phase transition. Nat. Mater. 10 693-697 (2011).

\section{cc) (i)}

This work is licensed under a Creative Commons Attribution 4.0 International License. The images or other third party material in this article are included in the article's Creative Commons license, unless indicated otherwise in the credit line; if the material is not included under the Creative Commons license, users will need to obtain permission from the license holder to reproduce the material. To view a copy of this license, visit http:// creativecommons.org/licenses/by/4.0/

(C) The Author(s) 2017

Supplementary Information accompanies the paper on the NPG Asia Materials website (http://www.nature.com/am) 